\title{
Blood flow to the corpus luteum-bearing ovary and ipsilateral uterine horn of cows during the oestrous cycle and early pregnancy
}

\author{
S. P. Ford* and J. R. Chenault $\dagger$ \\ Roman L. Hruska U.S. Meat Animal Research Center-Agricultural Research, Science and \\ Education Administration, U.S. Department of Agriculture, Clay Center, Nebraska 68933, \\ U.S.A.
}

\begin{abstract}
Summary. Total ovarian blood flow of cows (sum' of flows through the ovarian artery and uterine branch of the ovarian artery), measured by electromagnetic flow probes, followed a consistent cyclic pattern, being highest from Day 10 of the oestrous cycle (oestrus $=$ Day 0 ) until 4 days before the subsequent oestrus. During this period of high ovarian blood flow, blood of uterine origin flowed to the ovary through the uterine branch of the ovarian artery and was found to comprise $20-40 \%$ of the ovarian blood flow. Between Days -4 and -3 , ovarian blood flow dropped $(P<0.01)$ from $3.2 \pm 0.5$ to $1.0 \pm 0.4 \mathrm{ml} / \mathrm{min}$, coincident with a reversal of flow through this anastomosis. Uterine arterial blood flow was highest $(P<0.05)$ from 1 day before oestrus to the day of oestrus when compared to the remaining days of the oestrous cycle. Throughout the oestrous cycle, ovarian blood flow was positively correlated with systemic concentrations of progesterone $(r=0.55, P<0.01)$ and negatively correlated with systemic concentrations of oestradiol-17 $\beta(r=-0.42$, $P<0.01$ ). In contrast, uterine arterial blood flow was negatively correlated with systemic progesterone $(r=-0.25, P<0.05)$ and positively correlated with systemic oestradiol $(r=0.45, P<0.05)$. These data provide direct evidence in the cow that a portion of the blood flowing to the ovary containing a fully functional corpus luteum is contributed by the ipsilateral uterine artery.
\end{abstract}

\section{Introduction}

In 1962, Hutchinson observed a communicating link between the middle uterine artery and the adjacent ovarian artery in the gravid uterine horn of the cow. When a radiopaque medium was injected into the uterine artery of excised reproductive tracts, it crossed freely into the adjacent ovarian artery and into the ovary bearing the corpus luteum (CL). Corrosion casts of uterine and ovarian arteries demonstrated that the bovine ovary is supplied by the ovarian artery which divides before entering the ovary into an ovarian branch which supplies the ovary, broad ligament and infundibulum, and a uterine branch which supplies the tip of the horn, the oviduct and also anastomoses with a primary branch of the uterine artery in the mesometrium of the ipsilateral uterine horn (Lamond \& Drost, 1974). In addition to verifying the presence of an anastomotic link between a major branch of the uterine artery and the adjacent ovarian artery,

\footnotetext{
* Present address: Department of Animal Science, 11 Kildee Hall, Iowa State University, Ames, lowa 500111, U.S.A.

$\uparrow$ Present address: The Upjohn Company, Reproduction and Physiology, 9670-190-1, Kalamazoo, Michigan 49001, U.S.A.
} 
Lamond \& Drost (1974) reported that the anastomosis was larger on the side ipsilateral to the ovary containing a functional CL and that section of the uterine branch of the ovarian artery resulted in abnormal cyclic ovarian function. Lamond \& Drost (1974) suggested that the blood supply to the ovary containing the CL might be comprised of blood from both the uterine and ovarian arteries.

The present experiment was conducted to determine the source and quantity of blood supplying the luteal ovary of the cow during the oestrous cycle and early pregnancy. The correlations of uterine and ovarian blood flow with concentrations of oestradiol-17 $\beta$ and progesterone in systemic blood were also determined.

\section{Materials and Methods}

\section{Measurement of uterine and ovarian blood flow}

Three multiparous Hereford cows which had exhibited 3 consecutive oestrous cycles of normal duration (18-23 days) were used. Before surgery, the cows were stanchioned daily from 08:00 to 17:00 h for at least 3 weeks to adjust them to handling and confinement. A bull was used to check oestrus twice daily at 07:00 and 17:00 h throughout the experiment. Food and water were denied for $24 \mathrm{~h}$ before surgery which was performed on Day 10 of an oestrous cycle (day of oestrus $=$ Day 0 ). Procedures for induction of general anaesthesia were identical to those described by Ford, Chenault \& Echternkamp (1979).

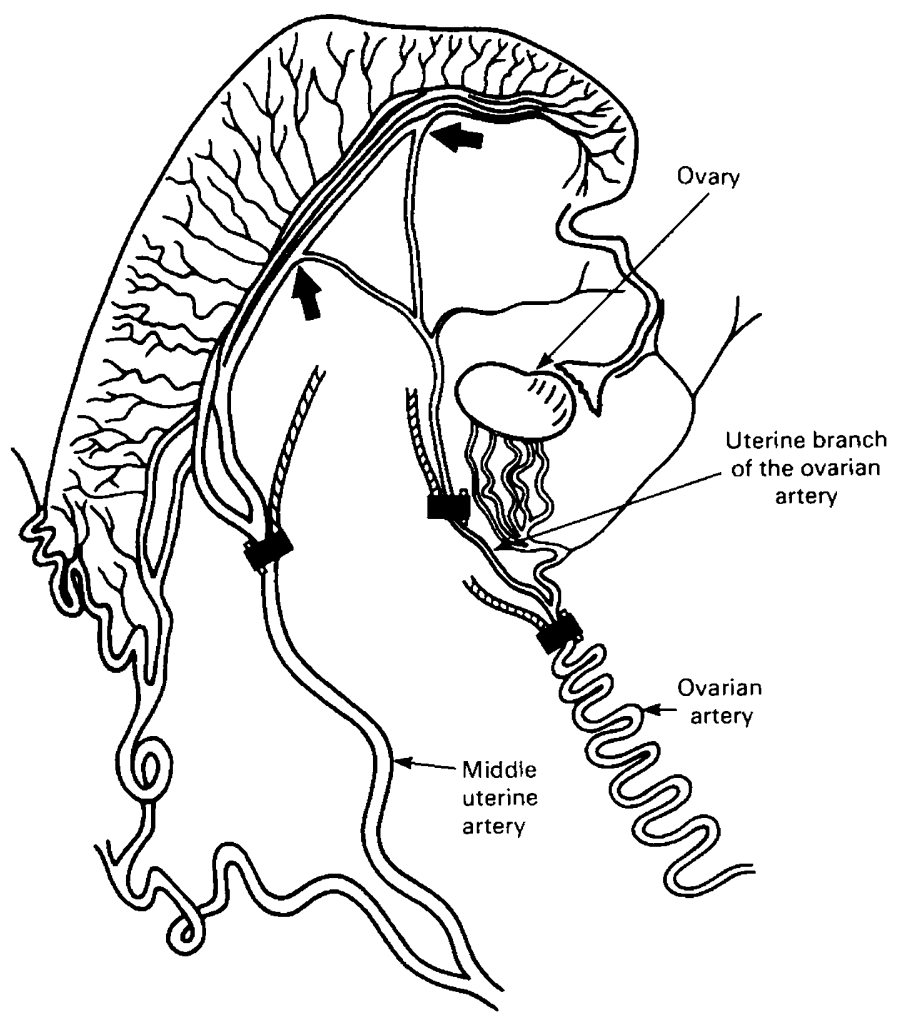

Text-fig. 1. Schematic drawing of the unilateral uterine and ovarian vasculature of the cow. Sites of placement of blood flow transducers ( $\boldsymbol{\square}$ ) are indicated and the functional anastomotic link between the uterine and ovarian arteries is arrowed. 
The uterus was exposed through a mid-ventral incision and the ovary not containing the CL was surgically removed. The adventitia was dissected from $1 \mathrm{~cm}$ segments of the ovarian artery and its uterine branch and the middle uterine artery on the side ipsilateral to the ovary which contained the CL and the vessel diameters were measured with a pair of calipers. Electromagnetic flow transducer probes of appropriate luminal diameters were passed through a small flank incision into the abdominal cavity, placed around the arteries, and fixed within the broad ligament with a series of silk sutures as illustrated in Text-fig. 1. The electrical connector for each of the 3 probes, used for attachment to the electromagnetic flow meter (Model 501D, Carolina Medical Electronics, Inc., King, North Carolina), was sutured to an elastic patch which was glued to the flank over the site of entry.

After surgery, the daily stanchioning routine was continued. Beginning on the second day after placement of the probes (i.e. Day 12 of the cycle), the direction and magnitude of blood flow through the 3 vessels on the side of the remaining ovary were each monitored continuously for periods of $15 \mathrm{~min}$. Blood flow values displayed by the flow meter at $15-\mathrm{sec}$ intervals during each daily 15 -min monitoring period were recorded and the values were averaged over the 15 -min periods for each artery to give the estimated blood flow for that day. The allocation of cows to the order of monitoring and the order of arterial monitoring for each cow was changed randomly each day. For ease of discussion, the flow of blood through the middle uterine artery will be referred to as uterine arterial blood flow and blood flow through the ovarian artery as ovarian arterial blood flow. The sum of the flow through the ovarian artery and its uterine branch will represent the total ovarian blood flow unless the flow in the uterine branch was in the direction of the uterus when it was subtracted from the value for the ovarian artery.

At the second oestrus after surgery, 2 of the cows were artificially inseminated at 12 and $24 \mathrm{~h}$ after the first signs of behavioural oestrus. On Day 18 after mating, the cows were slaughtered to verify pregnancy and the position of the flow transducers. The third cow did not exhibit a second oestrus and was slaughtered 36 days after the first oestrus after surgery. The CL appeared to be normal and the progesterone profile suggested that the cow had ovulated during this 36 -day period.

\section{Ovarian steroid measurements}

A jugular cannula, inserted at the time of surgery, was used to obtain blood from each cow immediately after monitoring of blood flow for determination of serum progesterone and oestradiol- $17 \beta$. Serum concentrations of progesterone were determined throughout the oestrous cycle. Oestradiol- $17 \beta$ concentrations were determined only from Day -6 to oestrus, to include the pro-oestrous rise observed previously (Ford et al., 1979). Radioimmunoassay of progesterone was a modification of a procedure described previously by Abraham, Hopper, Tulchinsky, Swerdloff \& Odell (1971) and utilized the same, fully characterized antiserum (S-49 No. 6). Although the same antiserum was used, blood serum was extracted with heptane instead of diethyl ether to improve recovery and chromatography was omitted. Sensitivity of the assay was defined as the amount of progesterone standard which yielded $95 \%$ of the counts/min in the buffer control tubes; this amount ranged from 5 to $7.8 \mathrm{pg}$. With this method, the blank value for serum from a cow on the day of oestrus was $0.40 \pm 0.03 \mathrm{ng} / \mathrm{ml}$ (s.e.m., $n=4$ ). The precision and accuracy of the procedure were evaluated by adding $0.05(n=4), 0.13(n=4), 0.25$ $(n=4,0.50(n=4), 1.25(n=4), 2.50(n=4)$ and $5.00(n=4)$ ng progesterone to this serum pool on a $\mathrm{ng} / \mathrm{ml}$ basis. The resulting progesterone concentrations ( \pm s.e.m.), after subtraction of the serum blank, were $0.05 \pm 0.01,0.13 \pm 0.01,0.25 \pm 0.01,0.41 \pm 0.02,1.04 \pm 0.02$, $2.49 \pm 0.05$ and $4.88 \pm 0.05 \mathrm{ng} / \mathrm{ml}$, respectively. Within-assay variability was determined from replicates $(n=10)$ of a serum pool from luteal-phase cows. The resulting concentration $( \pm$ s.e.m.) was $1.94 \pm 0.02 \mathrm{ng} / \mathrm{ml}$ (coefficient of variation $=2.9 \%$ ). Between-assay variation 
determined by assaying duplicates of the serum pool from luteal-phase cows in each assay $(n=8)$ was $5 \cdot 9 \%$.

Quantitation of oestradiol was a slight modification of a procedure previously reported by Echternkamp \& Hansel (1973) which utilized column chromatography to separate and purify oestradiol-17 $\beta$ before radioimmunoassay. In contrast with the previous methodology, $2 \mathrm{ml}$ serum were extracted twice with 2.5 volumes of benzene and the final benzene extract was washed once with 1 volume of deionized water to remove any water-soluble contaminants prior to chromatography. The antiserum (S-1554 No. 6) was obtained commercially from Dr G. E. Abraham (Division of Reproductive Biology, Harbor General Hospital, 1000 West Carson Street, Torrance, California 90509). The antiserum was very specific for oestradiol-17ß, exhibiting little cross-reactivity with oestrone $(0.6 \%)$, oestriol $(<0.01 \%)$ or testosterone $(<0.01 \%)$. Sensitivity of the assay, defined as the amount of oestradiol-17 $\beta$ standard which yielded $95 \%$ of the counts $/ \mathrm{min}$ in the buffer control tubes, ranged from 1.8 to $2.3 \mathrm{pg}$. The precision and accuracy were evaluated by adding $10,20,40,50$ and $80 \mathrm{pg}$ oestradiol-17 $\beta$ to charcoal-stripped plasma from an ovariectomized cow. After subtraction of the serum blank, the oestradiol concentrations $( \pm$ s.e.m.) were $10.0 \pm 0.6(n=4), 20.7 \pm 1.6(n=4), 45.0 \pm 2.9$ $(n=4), 58 \cdot 8 \pm 4.2(n=4)$, and $81.8 \pm 2.8(n=4)$, respectively. Within-assay variability, determined from duplicates, was $<20 \%$. Between-assay variation was determined by assaying duplicates of a pooled serum from oestrous cows and a pool of serum from cows during the luteal phase of the oestrous cycle in each assay $(n=6)$. Concentrations $( \pm$ s.e.m.) of oestradiol were $8.0 \pm 0.2 \mathrm{pg} / \mathrm{ml}$ for the pool of serum from oestrous cows and $4.3 \pm 0.3 \mathrm{pg} / \mathrm{ml}$ for the pool from luteal-phase cows.

\section{Statistical analysis}

Daily blood flow averages for each artery were considered as a single observation for statistical analysis to characterize time trends. Uterine and ovarian blood flow responses and changes in systemic concentrations of ovarian steroids were analysed by split-plot analysis of variance techniques and differences between means were tested for significance by the Bonferroni method (Kirk, 1968). Correlations between serum concentrations of progesterone and oestradiol- $17 \beta$ with uterine and ovarian blood flow were also determined.

Data from the cow that conceived at the second post-operative oestrus are excluded after that oestrus.

\section{Results}

Surgery and placement of blood flow transducers had no significant effect on oestrous cycle lengths exhibited by the cows in this study: immediately before surgery the mean \pm s.e.m. oestrous cycle length was $21.6 \pm 0.4$ days, while surgical and post-operative oestrous cycle lengths were $20 \cdot 0 \pm 1.8$ days $(N=5)$.

\section{Blood flow}

Uterine arterial blood flow was not measured in 1 of the 3 cows due to a faulty probe, and Text-fig. 2(a) therefore depicts the pattern of blood flow to the uteri of the remaining 2 cows. Uterine blood flow was highest $(P<0.05)$ on the day before oestrus and the day of oestrus when compared to the remaining days of the cycle. In addition, several transient increases in uterine arterial blood flow were observed during the luteal phase of the cycle in each cow. Total ovarian blood flow remained high $(3-4 \mathrm{ml} / \mathrm{min})$ until 4 days before oestrus then decreased $(P<0.01)$ abruptly (Text-fig. 2b). This precipitous decline in ovarian blood flow was achieved primarily by reversal of blood flow through the uterine branch of the ovarian artery in the direction of the 
uterus (Text-fig. 3). During the luteal phase, blood of uterine origin comprised $20-40 \%$ of the total ovarian blood flow.
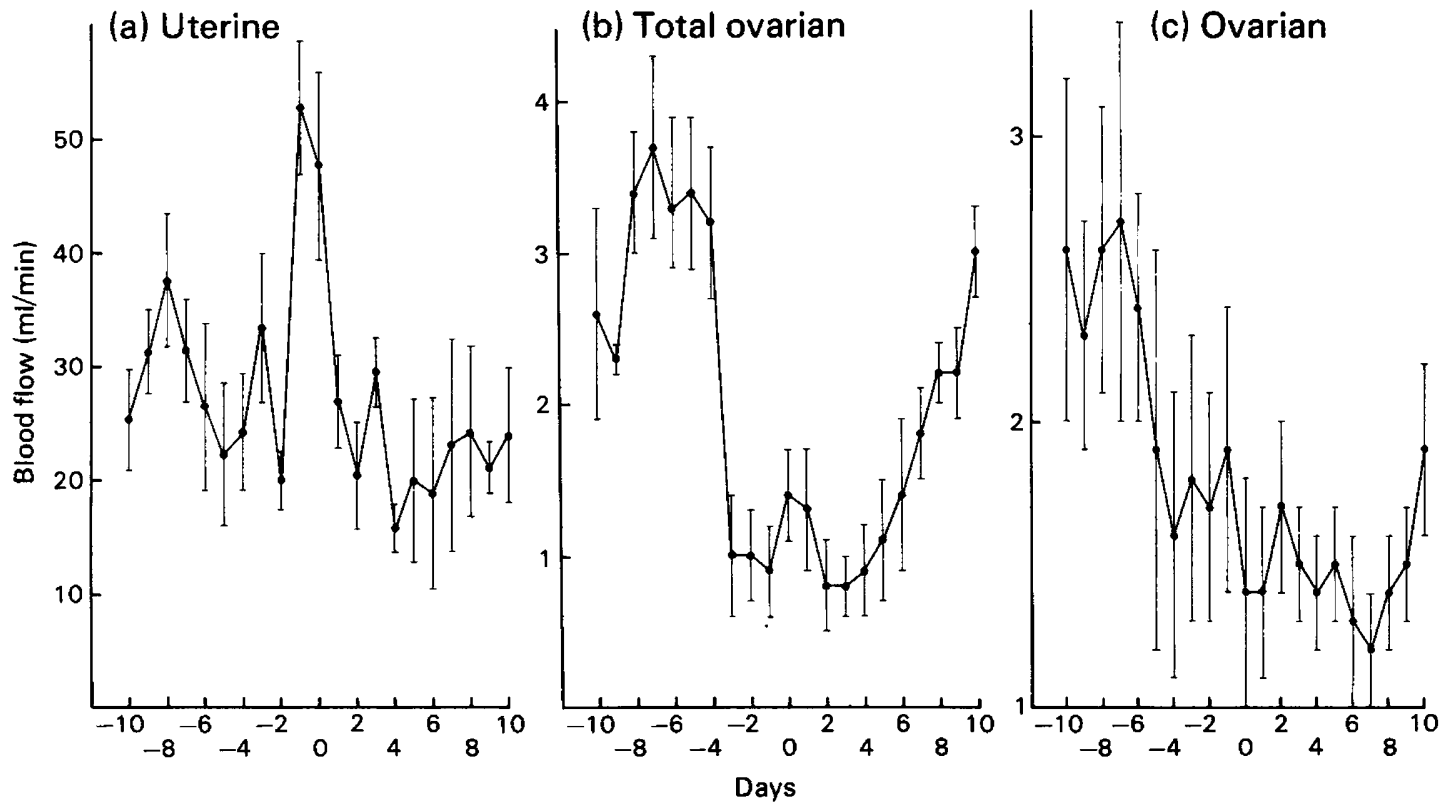

Text-fig. 2. Pattern of blood flow in non-pregnant cows throughout the oestrous cycle: (a) uterine arterial blood flow; (b) total ovarian blood flow; (c) ovarian arterial blood flow. In (a) each point represents the mean \pm s.e.m. of 4 observations until oestrus (Day 0) and 3 observations thereafter; in (b) and (c) the values are mean \pm s.e.m. for 5 observations before oestrus and 4 thereafter.

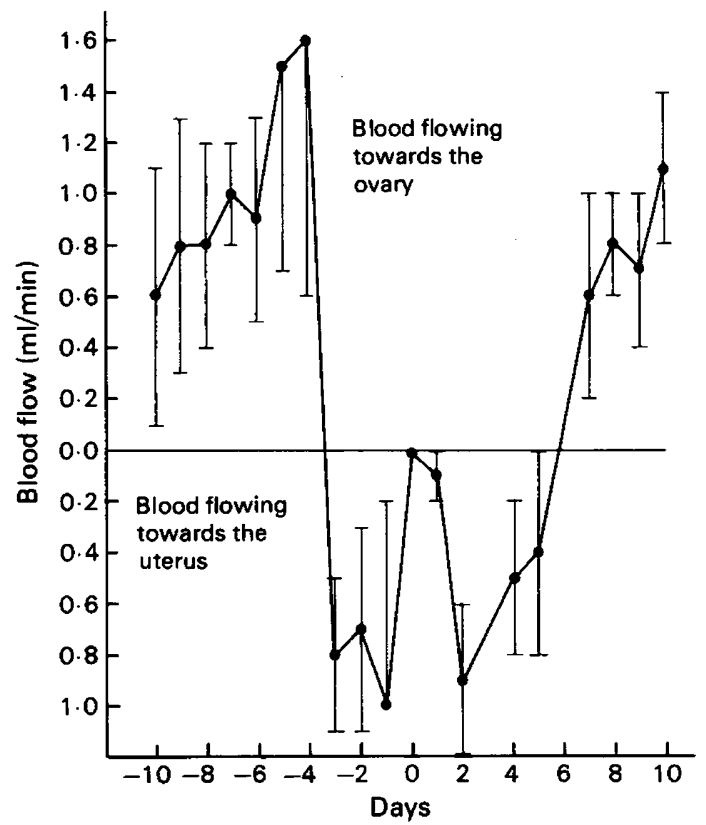

Text-fig. 3. Quantity of blood and direction of flow through the uterine branch of the ovarian artery before and after oestrus in non-pregnant cows. Values are mean \pm s.e.m. for 5 observations before oestrus (Day 0 ) and 4 observations thereafter. 


\section{Steroid hormones}

Progesterone concentrations in systemic blood exhibited a significant decline $(P<0.05)$ between 5 and 3 days before oestrus (Text-fig. 4). Concentrations of progesterone remained low until Day 4 and then increased to a high concentration by Day 10. Oestradiol-17 $\beta$ concentrations remained low between 6 and 3 days before oestrus, then increased $(P<0.05) 3$ to 4 -fold to reach peak values 1 day before oestrus.

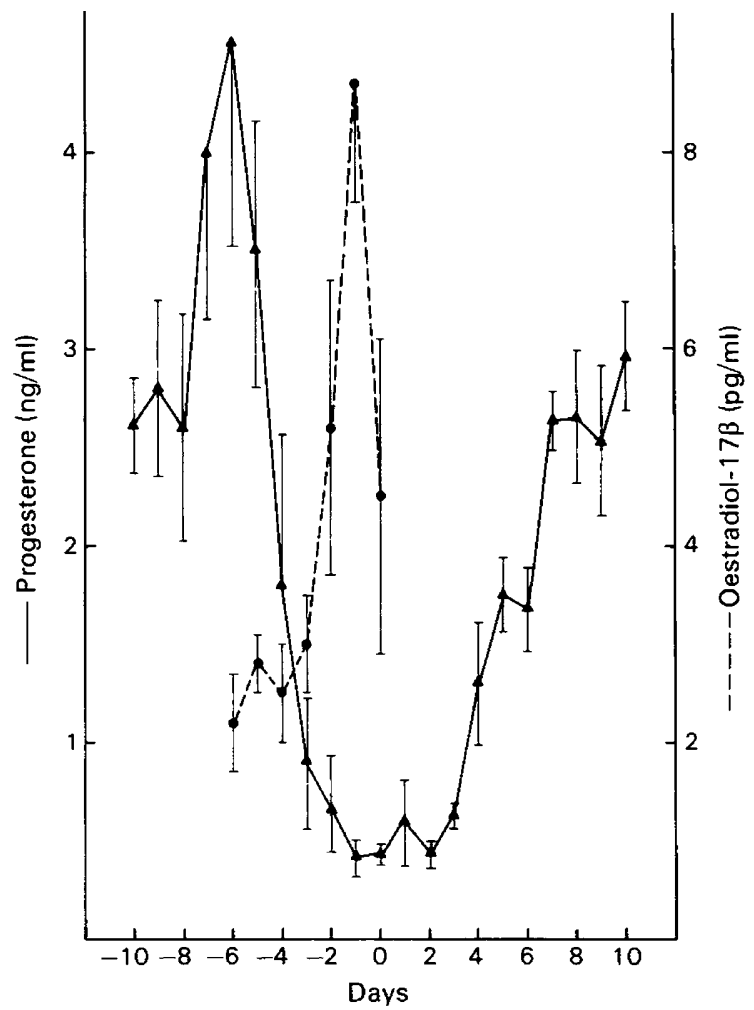

Text-fig. 4. Concentrations of progesterone (A) and oestradiol-17 $(\bullet)$ in systemic blood of non-pregnant cows during the oestrous cycle. Values are mean \pm s.e.m. for 5 observations before oestrus (Day 0 ) and 4 observations thereafter.

The correlations of blood flow with the systemic hormone concentrations are shown in Table 1. Total ovarian blood flow was positively correlated with systemic concentrations of progesterone $(P<0.01)$ and negatively correlated with oestradiol-17 $\beta(P<0.01)$. In contrast, uterine arterial blood flow was negatively correlated with systemic progesterone $(P<0.05)$ and positively correlated with oestradiol-17 $\beta(P<0.05)$ during similar periods in the oestrous cycle.

Table 1. Multiple correlations between concentrations of oestradiol-17 $\beta$ and progesterone in systemic blood and blood flow to the ovary bearing the corpus luteum and ipsilateral uterine horn

\begin{tabular}{|c|c|c|c|c|}
\hline Parameter & $\begin{array}{l}\text { Total ovarian } \\
\text { blood flow }\end{array}$ & $\begin{array}{l}\text { Ovarian arterial } \\
\text { blood flow }\end{array}$ & Progesterone & Oestradiol-17 $\beta \dagger$ \\
\hline $\begin{array}{l}\text { Uterine arterial blood flow } \\
\text { Total ovarian blood flow } \\
\text { Ovarian arterial blood flow } \\
\text { Progesterone }\end{array}$ & $0.22(63)$ & $\begin{array}{l}0.29(63)^{*} \\
0.52(105)^{* *}\end{array}$ & $\begin{array}{l}-0.25(63)^{*} \\
0.55(105)^{* *} \\
0.15(105)\end{array}$ & $\begin{array}{l}0.45(21)^{*} \\
-0.42(35)^{* *} \\
-0.09(35) \\
-0.35(35)^{*}\end{array}$ \\
\hline
\end{tabular}

${ }^{*} P<0.05 ; * * P<0.01$ (numbers in parentheses = degrees of freedom).

+ Correlations with oestradiol-17 $\beta$ were calculated only from Day -6 to oestrus (Day 0 ). 
Of the 2 cows which exhibited a second post-surgical oestrus and were inseminated, only one conceived. The pattern of uterine arterial blood flow was similar to that during the oestrous cycle until Day 16 when there was a 3-fold increase in blood flow to the gravid horn. Values then declined. This transient increase in one animal was coincident with a transient rise in total ovarian blood flow to the ipsilateral ovary which remained high until slaughter on Day 18.

\section{Discussion}

These data provide direct evidence that a significant portion $(20-40 \%)$ of the blood flow to the ovary containing a functional $\mathrm{CL}$ in the cow is supplied by the ipsilateral uterine artery through an anastomosis with the ovarian artery. However, the quantity of blood and the direction of flow through this anastomosis changed during the oestrous cycle, perhaps because of changes in uterine blood flow and (or) vascular resistance.

Uterine arterial contractility is greatest and uterine blood flow is least during the luteal phase of the oestrous cycle in cows (Ford, Weber \& Stormshak, 1976; Ford et al., 1979). Furthermore, Ford et al. (1976) determined that uterine arteries from the luteal side in unilaterally ovulating non-pregnant cows exhibited greater contractility in vitro in response to periarterial sympathetic nerve stimulation than did arteries from the contralateral side. These authors suggested that the greater contractility of uterine arteries supplying uterine horns ipsilateral to ovaries with a CL may result from exposure of these arteries to greatly increased progesterone concentrations in ovarian lymph (Lindner, Sass \& Morris, 1964; Morris \& Sass, 1966). The association between ovarian progesterone secretion and uterine arterial contractility was strengthened by the highly significant correlation $(r=0.72)$ found between uterine arterial contractility and concentrations of progesterone in systemic blood of ewes during the oestrous cycle (Ford et al., 1977). These studies are in agreement with present data which demonstrate a significant negative correlation between uterine arterial blood flow and systemic progesterone of cows throughout the oestrous cycle. Thus, an increased resistance to blood flow in the uterus during the luteal phase of the cycle may allow the uterine artery to participate in supplying blood to the CL-bearing ovary through the uterine branch of the ovarian artery. This flow would supplement the quantity of blood reaching the ovary through the ovarian artery at a time when the ovary requires a high rate of flow to maintain peak luteal function. During the ovine oestrous cycle, luteal progesterone secretion is highly correlated with blood flow through the CL (Niswender, Moore, Akbar, Nett \& Diekman, 1975).

The ovarian arterial flow increased from $1.4 \pm 0.5 \mathrm{ml} / \mathrm{min}$ at oestrus to $2.7 \pm 0.7 \mathrm{ml} / \mathrm{min}$ during the luteal phase of the oestrous cycle (Text-fig. 2c). The pattern of blood flow through the ovarian artery observed in this study is similar to results of Niswender et al. (1975) who demonstrated a decreased flow of blood to the ovary with the CL during the late luteal phase of the ewe with flow-rates being minimal at the subsequent oestrus.

The importance of the uterine contribution of blood to luteal function in this study is emphasized by the significant correlation $(r=0.55 ; P<0.01)$ between total ovarian blood flow and concentrations of progesterone in systemic blood throughout the oestrous cycle. In contrast, ovarian arterial blood flow was not significantly correlated with systemic concentrations of progesterone. The reversal of flow through the uterine branch of the ovarian artery in the direction of the uterus may result from the dilatatory effect of oestradiol on the bovine uterine vascular bed (Roman-Ponce, Thatcher, Caton, Barron \& Wilcox, 1978); there was a significant positive correlation between uterine arterial blood flow and systemic oestradiol-17 $\beta$ concentrations from 6 days before until the day of oestrus. In addition, total ovarian blood flow was negatively correlated with oestradiol-17 $\beta$ during the same period. Total ovarian blood flow in the 1 cow which conceived in this study remained high for the first 18 days of pregnancy. In 
addition, the increase in blood flow to the gravid uterine horn that was observed on Day 16 after mating is in agreement with our previous data (Ford et al., 1979).

The direction of blood flow in the utero-ovarian arterial anastomoses and the relative uterine and ovarian arterial contribution to the blood supply of the ovary during the oestrous cycle and pregnancy may be involved in local transfer mechanisms (Ginther, 1974) related to CL function. Wehrenberg, Chaichareon, Dierschke, Rankin \& Ginther (1977) observed that the uterus supplies $91-100 \%$ of the blood to ovaries of non-pregnant and early pregnant monkeys while during late pregnancy the ovarian artery becomes the dominant source of blood to the ovaries. These studies indicate the necessity of determining the relative contributions of the uterine and ovarian arteries when studying phenomena involving ovarian blood flow.

We thank Bill McDonald for technical assistance; Walt Green and Gary Peterson for care of experimental animals and help with the daily monitoring of blood flow; and the Agricultural Experiment Station, University of Nebraska, for co-operation.

Mention of a trade name, proprietary product, or specific equipment does not constitute a guarantee or warranty by the U.S. Department of Agriculture and does not imply its approval to the exclusion of other products that may be suitable.

\section{References}

Abraham, G.E., Hopper, K., Tulchinsky, D., Swerdloff, R.S. \& Odell, W.D. (1971) Simultaneous measurement of plasma progesterone, 17-hydroxyprogesterone and oestradiol-17 $\beta$ by radioimmunoassay. Analytical Letters 4, 325-335.

Echternkamp, S.E. \& Hansel, W. (1973) Concurrent changes in bovine plasma hormone levels prior to and during the first postpartum estrous cycle. $J$. Anim. Sci. 37, 1362-1370.

Ford, S.P., Weber, L.J. \& Stormshak, F. (1976) In vitro response of ovine and bovine uterine arteries to prostaglandin $\mathrm{F}_{2} \alpha$ and periarterial sympathetic nerve stimulation. Biol. Reprod. 15, 58-65.

Ford, S.P., Weber, L.J. \& Stormshak, F. (1977) Response of ovine uterine arteries to nerve stimulation after perfusions of prostaglandin $F_{2} \alpha$, norepinephrine or neurotransmitter antagonists. Endocrinology 101, 659-665.

Ford, S.P., Chenault, J.R. \& Echternkamp, S.E. (1979) Uterine blood flow of cows during the oestrous cycle and early pregnancy: effect of the conceptus on the uterine blood supply. J. Reprod. Fert. 56, 53-62.

Ginther, O.J. (1974) Internal regulation of physiological processes through local venoarterial pathways: a review. J. Anim. Sci. 39, 550-564.

Hutchinson, L.R. (1962) The hemodynamics of the isolated pregnant uterus of the cow (Bos taurus). Ph.D. thesis, Iowa State University, Ames.

Kirk, R.E. (1968) Experimental Design-Procedures for the Behavioral Sciences. Wadsworth Publishing Co., Belmont, California.

Lamond, D.R. \& Drost, M. (1974) Blood supply to the bovine ovary. J. Anim. Sci. 38, 106-112.

Lindner, H.R., Sass, M.B. \& Morris, B. (1964) Steroids in the ovarian lymph and blood of conscious ewes. $J$. Endocr. 30, 361-376.

Morris, B. \& Sass, M.B. (1966) The formation of lymph in the ovary. Proc. $R$. Soc. $B$ 164, 577-591.

Niswender, G.D., Moore, R.T., Akbar, A. M., Nett, T.M. \& Diekman, M.A. (1975) Flow of blood to the ovaries of ewes throughout the estrous cycle. Biol. Reprod. 13, 381-388.

Roman-Ponce, H., Thatcher, W. W., Caton, D., Barron, D.H. \& Wilcox, C.J. (1978) Thermal stress effects on uterine blood flow in dairy cows. J. Anim. Sci. 46, 175-180.

Wehrenberg, W.B., Chaichareon, D.P., Dierschke, D.J., Rankin, J.H. \& Ginther, O.J. (1977) Vascular dynamics of the reproductive tract in the female rhesus monkey: relative contributions of ovarian and uterine arteries. Biol. Reprod. 17, 148-153.

Received 12 December 1980 